\title{
GERADE ERFOLGREICH BEENDET: DER KONGRESS IN GRAZ - DAS TAO DES BEWEGUNGSAPPARATES
}

\section{Sehr geehrte Frau Kollegin! \\ Sehr geehrter Herr Kollege!}

Gerade ging unser 12.TAO-Kongress zum Thema „Bewegungsapparat" zu Ende und ich darf Ihnen berichten, dass sich in dieser Thematik in den vergangenen Jahren viel getan hat. Die ohnehin schon effektive Therapie mittels Körper und Ohr-Akupunktur hat wesentliche Fortschritte erreicht durch Einbau traditionell chinesischerVerfahren wie Gua Sha, Schröpfen, Moxa, spezifischer Kräuter und vielem mehr. Wir können heute auf eine riesengroße $\mathrm{Pa}-$ lette an Therapiemöglichkeiten zurückgreifen.

Schmerzen des Bewegungsapparates sind wohl die Hauptindikation für Akupunktur in unseren Ordinationen. Sie gelten laut WHO als der Bereich, in dem die Akupunktur als Mittel der ersten Wahl eingesetzt werden kann. Und doch ist die Therapie oft nicht so erfolgreich, wie wir es uns wünschen würden.

Dr. Aluani und ich haben nun den TAO-Kongress zum Anlass genommen, um eine völlig neue Diplomausbildung zu schaffen, da wir die vielen neuen und traditionellen Methoden einmal zusammengefasst vorstellen wollen. In unseren Ordinationen bewährt sich dieses ,integrative“ Vorgehen, in dem mehrere Therapiemethoden individuell auf den konkreten Patienten hin abgestimmt und dann koordiniert angewendet werden. Wir sehen, dass so Erfolge erzielt werden, die mit einer einfachen Akupunktur vor allem in ihrer Nachhaltigkeit oft nicht möglich sind. Diese Kursserie wird Abhilfe bei Problemfällen schaffen und die Erfolgsquote bei Beschwerden des Bewegungsapparates noch wesentlich anheben. Denn es wird gezeigt, wie unterschiedlichste Therapieoptionen kombiniert eingesetzt werden. So reicht die Bandbreite der Themen von einer Wiederholung der wichtigsten anatomischen Grundlagen bis hin $\mathrm{zu}$ den Therapiemaßnahmen, die sich in unseren Ordinationen seit vielen Jahren im praktischen Einsatz bestens bewährt haben.
Der Aufbau und die Funktion der Gelenke, der wichtigsten Muskeln und Faszien werden besprochen, die Körperund Ohrakupunktur wiederholt und vertieft, manuelle Untersuchungstechniken erarbeitet und schließlich so unterschiedliche Therapien wie Akupunktur, Infiltration, Triggerpunkttechniken und Kinesio-Taping vorgestellt. Als besondere Schwerpunkt-Themen haben wir vorgesehen: Die Liquid Acupuncture, eine Injektionstechnik, bei der in die betroffenen Ohrareale homöopathische Arzneien injiziert werden und die oft innerhalb von Minuten eine Schmerzreduktion bewirkt, die Therapie mittels Verum-Laser, die neben völliger Schmerzfreiheit starke Effekte erzielen kann und vieles mehr.

Wie bei allen unseren Kursen üblich, wird der Stoff nicht nur in der Theorie vorgestellt, sondern auch geübt und praktiziert. Wir wollen, dass Sie bereits in den Tagen nach dem Kurs Ihr neuerworbenes Wissen korrekt an Ihren Patienten anwenden können.

Als Bestätigung der besonderen Kompetenz in Sachen Bewegungsapparat erhalten alle AbsolventInnen unser „Spezialdiplom für Integrative Therapie des Bewegungsapparats" überreicht.

Diese Kursserie wird 2016 erstmals angeboten. Wir werden Sie noch mit einem speziellen Folder über diese Diplomausbildung informieren. Bei Interesse können Sie auch jederzeit bei uns anrufen, wir beraten Sie gerne.

So wünsche ich Ihnen noch einen schönen Sommerausklang und sende Ihnen beste, kollegiale Grüße Ihr

Prof. Dr. med. Leopold Dorfer

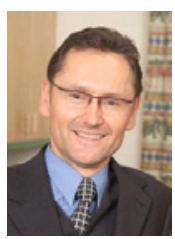

Prof. Dr. med. Leopold Dorfer

Präsident der Österreichischen Gesellschaft für Kontrollierte Akupunktur und TCM (OGKA)

Glacisstraße 7, A-8010 Graz

Tel. $+43316 / 374050$

E-Mail: office@ogka.at, Internet:www.ogka.at 